\title{
Physical Integrity of 3D Printed Parts for use as Embossing Tools
}

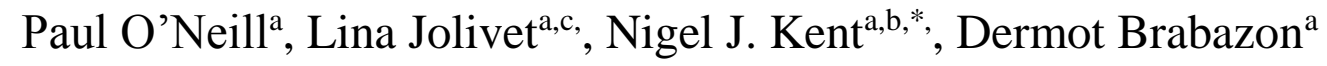

${ }^{a}$ Advanced Processing Technology Research Centre, Dublin City University, Ireland.

${ }^{b}$ School of Mechanical and Design Engineering, Dublin Institute of Technology.

${ }^{c}$ Institut National des Sciences Appliquées de Lyon, France.

Corresponding author*:

Dr. Nigel J. Kent.

Rm387,

Dublin Institute of Technology,

Bolton St,

Dublin 1.

Tel: +35314023885

Email: nigel.kent@dit.ie 
On inception, 3D printed parts were typically used at prototyping stage to give the end user/customer a real world concept of how the part may appear when traditional manufacturing techniques were employed for final part fabrication. In this context, mechanical properties such as load bearing capacity or wear rate were not typically of primary concern. This paper investigates, given the advances in 3D printing technology, the potential for using 3D printed parts for high throughput embossing tools. The key mechanical properties for embossing tools are compression and wear rate. To this end, commercially available engineering grade photopolymer materials were characterised in terms of compression and wear using ASTM D695 and ASTM G99 standards respectively. Parts were fabricated via the Polyjet ink-jetting 3D printing technique using the commercially available Connex 260 from Stratasys. Given the nature of the fabrication technique, differences in compressive strength of the material based on orientation of build were also investigated.

Keywords: PolyJet; 3D printing; Compressive Strength; Wear Rate; Ink-jetting; Embossing.

\section{Introduction.}

Additive manufacturing (AM) has evolved rapidly since the invention of stereolithography in 1986 by Charles Hull [1] to encompass a range of manufacturing techniques based on layer by layer fabrication. These techniques have become synonymous with the term '3D printing', a technique based on the principle that each part is made up of thousands of voxels, analogous to a pixel but in three dimensions instead of two. Each voxel has an $\mathrm{X}, \mathrm{Y}$ and $\mathrm{Z}$ dimension that is a function of the accuracy of the 3D printing hardware in the scanning $(\mathrm{X}, \mathrm{Y})$ and build $(\mathrm{Z})$ directions. There are a wide range of 3D printing methods available which can be loosely grouped into three broad categories, fused deposition modelling, optical-lithography, and 
powder-bed techniques.

Traditionally, these techniques have only been used for applications in design and prototyping at the preliminary stage of the product design life cycle. However, recent advances in 3D printing technologies have led to an increase in build speed, resolution, accuracy, and materials available, potentially making 3D printing a viable production method for a number of low-volume high-value industries such as aerospace, automotive, production tooling, jewellery, dentistry, and audiology [2]. This move toward direct manufacturing of fully functional components has been termed the 'third industrial revolution' [2-5]. This move represents a huge opportunity for modern manufacturers who rely largely on digital processes and where tooling represents the only off-line element in the process chain. In this respect, 3D printing can be used to digitise this final process element.

While 3D printing presents a huge opportunity, there is a significant challenge in overcoming the drawbacks associated with the production methods. Ensuring that the finished product can withstand the structural loading and wear conditions common in everyday use is inherently more complex when dealing with a 3D printed part. Material properties, for example, are difficult to predict due to the anisotropy of the layered structure and depend heavily on process parameters such as part orientation, layer height, and material type [6]. This is particularly important for applications in rapid tooling and medical implants where design requirements are more rigorous and where there has been a huge uptake in additive manufacturing techniques due to their ability to provide custom, low-volume parts at lower cost.

Among the current commercially available 3D printing technologies photopolymer based inkjet printers (i3DP) show significant promise with regard to build speed, resolution, and material selection [7]. The technique involves jetting droplets of 
UV curable resin and a wax or gel-like support structure and instantly curing under UV layer of photocurable polymer onto the print bed in a process similar to the standard 2D inkjet printing process. This layer is then partially cured through irradiation with an ultraviolet light source. When this layer is complete, the print bed moves down in the ' $\mathrm{z}$ ' direction as indicated in Figure. 1 and the process is repeated until the part is complete. During printing, a wax or gel-like support material is deposited only in areas that contain hollow parts or overhangs and does not crosslink with the main build material. This support material is removed after printing by heating (in the case of the waxy material (3D Systems MultiJet Printing)), or by using a high pressure water jet, or by sonication in a bath of sodium hydroxide solution (in the case of the gel-like material (Stratasys PolyJet Technology)) in a process called part development [9].

Resolution of the i3DP process depends on the DPI, or dots-per-inch, of the print head. Modern printers like the Objet Connex ${ }^{\mathrm{TM}}$ or the 3D systems Projet ${ }^{\mathrm{TM}} 3500 \mathrm{can}$ achieve a layer resolution down to $16 \mu \mathrm{m}$ with a DPI of 600-750 in the horizontal plane. The Objet range of 3D printers from Stratasys represent the forefront of i3DP with the capability to print a selection of 14 proprietary materials in single- and multi-material modes. The entry level Connex 1 , used in this work, typifies the PolyJet process and, as such, represents an ideal baseline for investigations into the processing capabilities of the PolyJet process.

Here we investigate the effect of build orientation on material properties including yield strength, compressive strength, elastic modulus, strain, and wear, using a commercially available PolyJet 3D printer, the Objet 260 Connex 1 from Stratasys. Build orientation is a key design input in additively manufactured parts due to the anisotropic nature of the build process and can result in a relatively large variation in 
mechanical properties between orientations. This relationship has been studied previously in respect to tensile stress [10-13], surface roughness [10], and thermomechanical properties [14].

\section{Materials and Methods}

\section{Test Pieces.}

All test parts for this work were based on guideline from respective ASTM standards $[15,16]$. These parts were generated in SolidWorks 2014-2015 and saved in Standard Tessellation Language (STL) format for subsequent transfer to an Objet 260 Connex 1 commercial 3D printer from Stratasys. STL files are generated by tessellating the surface of the specified part into a series of small triangles. On saving the part, custom STL parameters were used in an effort to enhance part quality. The 'deviation' parameter used to control whole part tessellation, influencing whole part accuracy, was changed from $0.02 \mathrm{~mm}$ to $0.0025 \mathrm{~mm}$. The 'angle' parameter, influencing smaller detail tessellation was modified from $10 \mathrm{deg}$ to $0.5 \mathrm{deg}$. These modifications, representing the best available through SolidWorks, had the net effect of significantly increasing the file size for the parts, typically on the order of a ten-fold increase. These modifications had a negligible effect on actual build times of the parts while ensuring maximum dimensional accuracy was achieved in printing.

In total, three materials from the PolyJet range were tested:

- Vero White RGD835 is among the more established polyjet materials and is closely aligned to Acrylonitrile Butadiene Styrene (ABS) and High-Impact Polystyrene (HIPS) in terms of properties. RGD835 falls into the Stratasys 'standard category' of plastics. 
- High Temp RGD 525 has a higher glass transition temperature (65 deg C) than

A summary of the wear and compression test specimen dimensions is shown in Table 1.

\section{Compression Testing.}

Compression testing was carried out based on ASTM D695 - Standard Test Method for Compressive Properties of Rigid Plastics [15]. Cylindrical parts, each of dia $12.7 \mathrm{~mm}$ and $25.4 \mathrm{~mm}$ long, were printed in each of the materials described. In order to investigate the effect of print direction on the compressive properties of a printed part, the test piece was printed in three orthogonal directions. The cylindrical test piece was printed with its longitudinal axis; in parallel with direction of the print head traverse direction ( $\mathrm{X}$ direction Figure1), perpendicular to the print head traverse direction ( $\mathrm{Y}$ direction Figure1) and normal to the print bed ( $\mathrm{Z}$ direction Figure 1). Four test samples were printed for each material and print orientation under investigation.

Compressive tests were carried out using a Zwick/Roell Z050 universal testing machine. The Zwick universal tester is fitted with a $50 \mathrm{kN}$ load cell and each sample was compressed at a cross head speed of $1.3 \mathrm{~mm} / \mathrm{min}$ until failure of the part, as per ASTM D695 [15]. Companion software TestXpertII was employed to capture and record Force in $\mathrm{kN}$ against $\%$ deformation of the part. Using the data obtained from the Zwick 
universal testing machine, four parameters were determined for each material, Modulus of Elasticity, Yield Strength, Maximum Compressive Strength and Strain at Maximum Compressive Strength.

\section{Wear Testing.}

Wear tests were carried out based on ASTM G99 - Standard Test Method for Wear Testing with a Pin-on-Disk Apparatus [16]. The schematic for the pin and disk apparatus is seen in Figure 2 (A). A pin is placed, under a known load, in contact with the sample of interest at a known distance from the centre of rotation of the test sample. The sample is then rotated at a defined speed for a defined number of revolutions. Given that the distance from the pin to the centre of rotation is known, the overall distance of travel of the pin on the surface can be calculated. Based on the weight of the part pre- and post-testing, the amount of material removed during testing can be calculated and the wear rate between the two materials determined. Figure 2 (B) shows an image of the actual experimental rig. The experimental rig was custom developed for wear testing purposes and consists of a 3mm diameter flat steel (63-65 HRC) pin, capable of being positioned across a range of radii, under varying loads, for a defined number of revolutions given by the revolution counter as indicated in Figure 2 (B).

In terms of testing the photopolymer materials, disks of diameter $45 \mathrm{~mm}$ and of thickness $3 \mathrm{~mm}$ were printed in each material. The flat cylindrical pin diameter was in contact with the sample surface, $15 \mathrm{~mm}$ from the centre of rotation of the test piece, under a load of $24.72 \mathrm{~N}$. The sample was rotated at $150 \mathrm{rpm}$ for 2000 seconds giving a total number of 5000 revolutions.

Following the testing procedure, large debris was removed using compressed air. The part was then submerged in Acetone, placed in an ultrasonic bath for 15 minutes and subsequently dried using compressed air to remove any smaller remaining 
debris. For each material four samples were tested in order to determine the specific wear rate of the material.

Following experimental testing, volume loss could be determined using:

$$
\text { Volume loss }\left(\mathrm{mm}^{3}\right)=\frac{\text { Mass } \operatorname{loss}(\mathrm{g})}{\operatorname{Density}\left(\mathrm{g} \cdot \mathrm{cm}^{-3}\right)} X 1000 .
$$

Mass loss of each material was determined via mass measurements of each disk before and after the experiment using a CP1245 balance from Sartorious. The polymerised densities of the samples were taken from material properties provided by Stratasys [17].

The specific wear rate of each sample was found using:

$$
\text { Specific Wear Rate, } K\left(\frac{\mathrm{mm}^{3}}{N . m}\right)=\frac{\operatorname{Volume} \operatorname{loss}\left(\mathrm{mm}^{3}\right)}{\operatorname{Load}(N) \times \operatorname{Sliding} \operatorname{Distance}(\mathrm{m})} \text {. }
$$

Where the sliding distance is calculated by using Eq. 3:

$$
\text { Sliding Distance }(\mathrm{mm})=\text { No. Revolutions } \times \text { Diameter of } \operatorname{track}(\mathrm{mm}) \times \pi \text {. }
$$

For all tests carried out, the sliding distance was determined to be $471.239 \mathrm{~m}$.

\section{Results}

\section{Compression.}

Figure 3 shows the results from compression testing of each material in each print orientation. There is no visual difference in results due to print orientation of the parts for both Vero White (Figure 3 (a-c)) and MED 610 (Figure 3 (d-f)). However, there is a marked difference between the compression results for Vero White/MED 610 and those of RGD525 (Figure 3 (h-i)). This marked difference in results in due to the fact, the 
Object 260 Connex 1 machine automatically generates an internal lattice structure within all parts printed in RGD525. The cavities within this lattice are filled with SUP705, the support material used in the Objet 260 Connex 1 machine. This lattice structure is visible in Figure 3 (j).

This setting is hard coded as part of the machine firmware and according to the application notes for RGD525 [18]:

'High-temperature parts are fabricated from a combination of two materials, RGD525 and SUP705 (the Support material). This unique combination enhances dimensional stability. However, drilling of printed parts is not recommended.'

Given that RGD525 is a member of the Stratasys 'engineering plastics' family, the inability to print solid parts in this material is seen as a limitation on use of this material for engineering purposes. Nonetheless compression results, based on ASTM D695 guidelines for compression testing of rigid plastics were carried out on all materials for comparative purposes. Compression results taken from the Zwick/Roell universal testing machine can be seen in Figure 4. The results are relatively consistent across all print orientations and agree with results previously obtained [19].

\section{Wear Testing.}

Post-test images of wear test samples can be seen in Figure 5. For each part a wear track is readily visible after 5000 revolutions. Given the internal lattice structure of RGD 525 printed parts previously described, care was taken during wear testing to ensure wear occurred only on the RGD525 surface and the internal lattice was not exposed. Using the Eqs (1-3) described previously, specific wear rate was calculated for each material. The mean and standard deviation of wear rate results can be seen in Figure 6 . 


\section{Discussion.}

The results from compression testing shown in Figure 4 can be seen to be largely independent of part orientation relative to print head traverse direction. There is minimal difference between part orientation in terms of compressive strength, yield strength, elastic modulus and strain values.

In terms of absolute values of the test parameters, values taken for parts printed in RGD 525 should not be taken as representative of RGD525 material only. As previously described, in an effort to enhance dimensional stability of the material, Stratasys automatically infuse parts printed in RGD525 with SUP705 support material. Given the nature of the support material, it was not an unexpected finding that these test parts printed in a SUP705-RGD525 hybrid, be significantly less robust than those printed only in RGD525 photopolymer. Nonetheless, the values recorded represent the compressive capacity of the designed part, with RGD525 as the selected material, printed using the Objet 260 Connex 1.

For both Vero White and MED610, the recorded Elastic Modulus was lower than that given by the manufacturers. The manufacturers state an Elastic Modulus range of 2-3 GPa for both materials [17] while results recorded using the Zwick/Roell recorded values of approximately $1.75-1.8 \mathrm{GPa}$ along all orientations. This variation in Elastic Modulus values may be attributed to the fact the manufacturers values are found from tensile testing while the experiments here were carried out under compressive loading.

Given the compressive strength properties between MED610 and VeroWhite are comparable; both these materials can be considered equally suitable for use in embossing tooling. The use of either of these materials as embossing tooling is based on the assumption the maximum compressive force of the embossing procedure does not exceed the limits of the material properties of the tool. 
From a specific wear rate perspective, RGD525 has the lowest mean wear rate across all materials indicating that RGD525 is a more suitable material than VeroWhite or MED610 for use in embossing tooling applications where the wear rate is perhaps more significant than compressive force.

\section{Conclusion}

For all materials tested, print orientation of the part relative to the print head traverse direction was found not to impact on compressive strength properties of the parts. Expectations from the manufacturer specification sheets [17] would indicate that RGD525 should be the most suitable material for use when printing embossing tools. However, given the apparent dimensional limitations printing with this material, resulting in the need to infuse the parts with SUP705, use of RGD525 exclusively for applications where compressive strength is a key consideration should be avoided.

In contrast to compressive testing of samples, specific wear rate testing is primarily a function of the part surface as opposed to the bulk material of the part. As a result, infusion of the bulk material became irrelevant and specific wear rate results for RGD525 were indicative of the specific wear rate for only RGD525 material. Specific wear test results indicate the preferred material for wear resistance is RGD525. Given that RGD525 is unsuitable for bulk printing for applications where compressive strength is a priority, there is capacity to exploit the wear resistance of RGD525, coupled with the bulk compressive strength properties of either VeroWhite or MED610 through the dual material print capacity of the Objet260 Connex1.

The Objet Studio software package allows the user to coat a part in a material different to that of the bulk material. Printing components in VeroWhite/MED610 and coating them in RGD252 should have a net result in maintaining the bulk compressive strength capabilities of the VeroWhite/MED610 while also having the specific wear rate 
capability of the RGD525. This presents an opportunity for further study in the case of a composite consisting of either VeroWhite or MED610 bulk material finished with a thin layer of RGD525.

\section{References.}

[1] C. W. Hull, "Apparatus for production of three-dimensional objects by stereolithography," U.S. Patent 4,575,3301986.

[2] Y. Huang, M. C. Leu, J. Mazumder, and A. Donmez, "Additive Manufacturing: Current State, Future Potential, Gaps and Needs, and Recommendations," J. Manuf. Sci. Eng., vol. 137, no. 1, p. 14001, Feb. 2015.

[3] The Economist, "Manufacturing: The Third Industrial Revolution," The Economist, 2012. [Online]. Available: http://www.economist.com/printedition/2012-04-21.

[4] S. Waheed, J. M. Cabot, N. P. Macdonald, T. Lewis, R. M. Guijt, B. Paull, and M. C. Breadmore, "3D printed microfluidic devices: enablers and barriers," Lab Chip, vol. 16, no. 11, pp. 1993-2013, 2016.

[5] H. Becker, "Hype, hope and hubris: the quest for the killer application in microfluidics.," Lab Chip, vol. 9, no. 15, pp. 2119-22, Aug. 2009.

[6] A. Kesy and J. Kotlinski, "Mechanical properties of parts produced by using polymer jetting technology," Arch. Civ. Mech. Eng., vol. 10, no. 3, pp. 37-50, Jan. 2010.

[7] M. Javaid, L. Kumar, A. Haleem, and V. Kumar, "Product Design and Development using Polyjet Rapid Prototyping Technology," Control Theory Informatics, vol. 5, no. 3, pp. 12-20, 2015.

[8] DREAMS, Material Jetting [Online], Available: http://www.me.vt.edu/dreams/material-jetting/ (accessed 28 Oct 2016)

[9] B. Y. Ang, C. K. Chua, and Z. H. Du, "Study of trapped material in rapid prototyping parts," Int. J. Adv. Manuf. Technol., vol. 16, no. 2, pp. 120-130, 2000.

[10] A. Cazon, P. Morer, and L. Matey, "PolyJet technology for product prototyping: Tensile strength and surface roughness properties," Proc. Inst. Mech. Eng. Part B J. Eng. Manuf., vol. 228, no. 12, pp. 1664-1675, 2014. 
[11] S. Adamczak, J. Bochnia, and B. Kaczmarska, "Estimating the uncertainty of tensile strength measurement for a photocured material produced by additive manufacturing," Metrol. Meas. Syst., vol. 21, no. 3, pp. 553-560, 2014.

[12] M. Sugavaneswaran and G. Arumaikkannu, "Modelling for randomly oriented multi material additive manufacturing component and its fabrication," Mater. Des., vol. 54, pp. 779-785, 2014.

[13] J. Mueller, K. Shea, and C. Daraio, "Mechanical properties of parts fabricated with inkjet 3D printing through efficient experimental design," Mater. Des., vol. 86, pp. 902-912, 2015.

[14] T. Tábi, N. K. Kovács, I. E. Sajó, T. Czigány, S. Hajba, and J. G. Kovács, "Comparison of thermal, mechanical and thermomechanical properties of poly(lactic acid) injection-molded into epoxy-based Rapid Prototyped (PolyJet) and conventional steel mold," J. Therm. Anal. Calorim., vol. 123, no. 1, pp. 349-361, 2016.

[15] ASTM D695-02a, Standard Test Method for Compressive Properties of Rigid Plastics, ASTM International, West Conshohocken, PA, 2002, www.astm.org

[16] ASTM G99-95a(2000)e1, Standard Test Method for Wear Testing with a Pinon-Disk Apparatus, ASTM International, West Conshohocken, PA, 2000, www.astm.org

[17] Stratasys, PolyJet Materials Data Sheet [Online], Available: http://usglobalimages.stratasys.com/Main/Files/Material_Spec_Sheets/MSS_PJ_ PJMaterialsDataSheet.pdf?v=635785205440671440 (accessed 26 Oct 2016)

[18] Stratasys, PolyJet Best Practice [Online], Available: http://www.advancedtek.com/wp-content/uploads/2016/08/Best_Practice__High-Temperature_RGD525_Model_Material_-_Web_English_11-15.pdf (accessed 26 Oct 2016)

[19] J. Kotlinski, "Mechanical properties of commercial rapid prototyping materials," Rapid Prototyp. J., vol. 20, no. 6, pp. 499-510, 2014. 
Figure 1. The inkjet 3D printing technique (Stratasys Objet). Liquid photopolymer and support material are deposited on to the printer bed and subsequently cured using an ultraviolet (UV) lamp [8].

Table 1. Test performed, American Society for Testing and Materials (ASTM) standard, and number of samples.

Figure 2. Pin on Disk apparatus. Figure 2 (A) shows the principle of pin on disk wear testing, a pin is placed on a rotating disk under load, the amount of material removed can be used to determine specific wear rate. Figure 2 (B) shows the experimental apparatus used for testing.

Figure 3. Compression testing results from all materials, in all print orientations. Figure 3. (a-c) shows Vero White printed along x,y,z axes respectively. Figure 3. (d-f) Med 610 along $x, y, z$ axes respectively and Figure 3 (g-i) show the results from RGD525 along the $\mathrm{x}, \mathrm{y}, \mathrm{z}$ axis respectively. Each image also shows an untested part for comparison. Figure 3. (j) shows the internal lattice structure used when printing solid parts using RGD525.

Figure 4. Compressive properties of tested materials determined from compression testing. Each graph shows results for all materials along three orthogonal axes (X, Y and Z) relative to the print head traverse direction. (A) Compressive Yield Strength (B) Max Compressive Strength (C) \% Strain at Failure and (D) Elastic Modulus of samples.

Figure 5. Representative samples post wear testing. For each sample a wear track is visible along the surface.

Figure 6. Wear rate results showing mean specific wear rate and standard deviations for all three materials tested. 


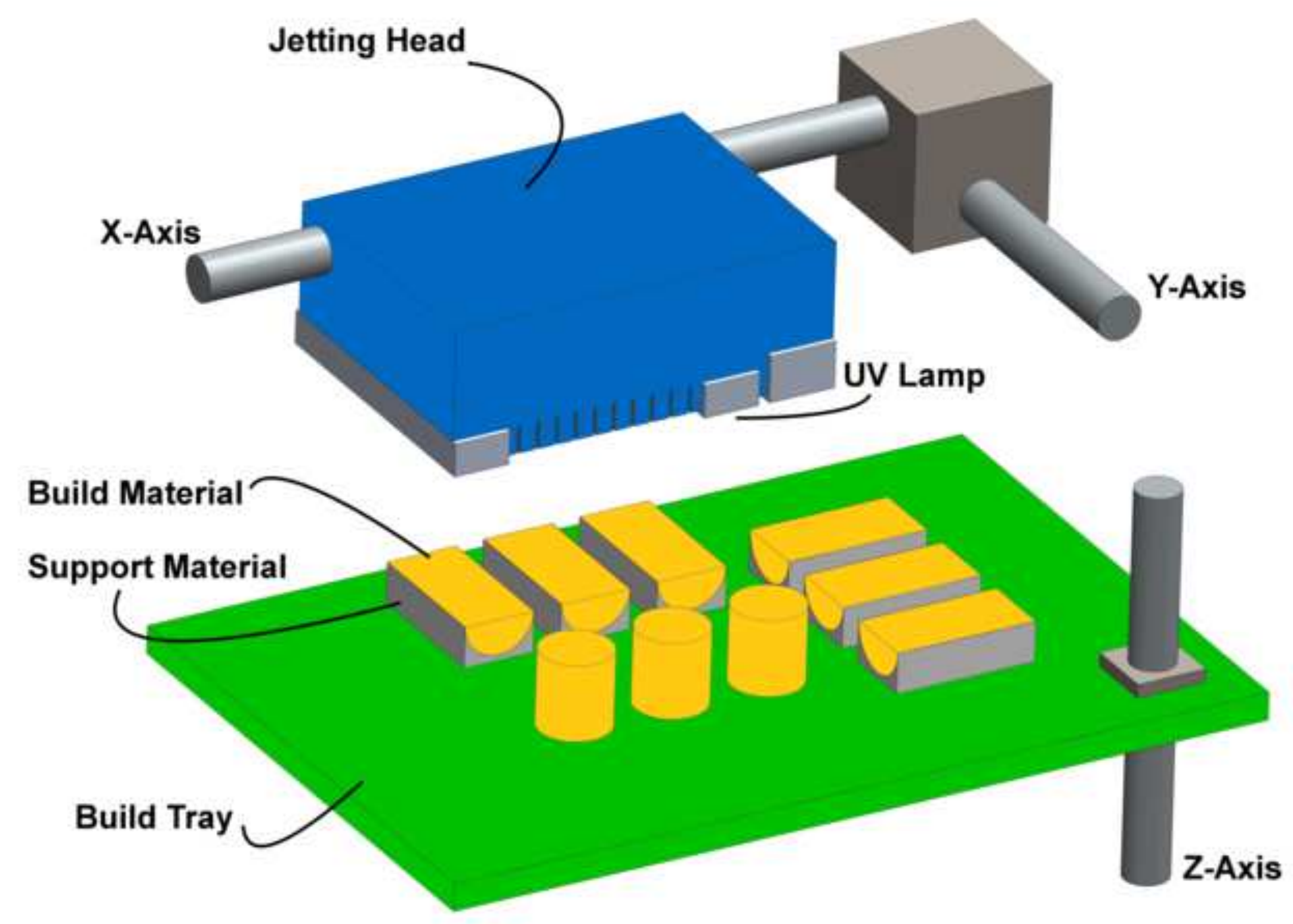


Test Specimen shape \& input dimensions (diameter $\mathrm{x}$ thickness)

Compression test Cylinder

(12.7 $\mathrm{mm} \times 25.4 \mathrm{~mm})$

4 in $\mathrm{x}$-direction

ASTM Standards D695 [15]

4 in y-direction

4 in z-direction

Wear test

Disk

4

(45 mm x $3 \mathrm{~mm})$

ASTM Standards G99 [16] 
A

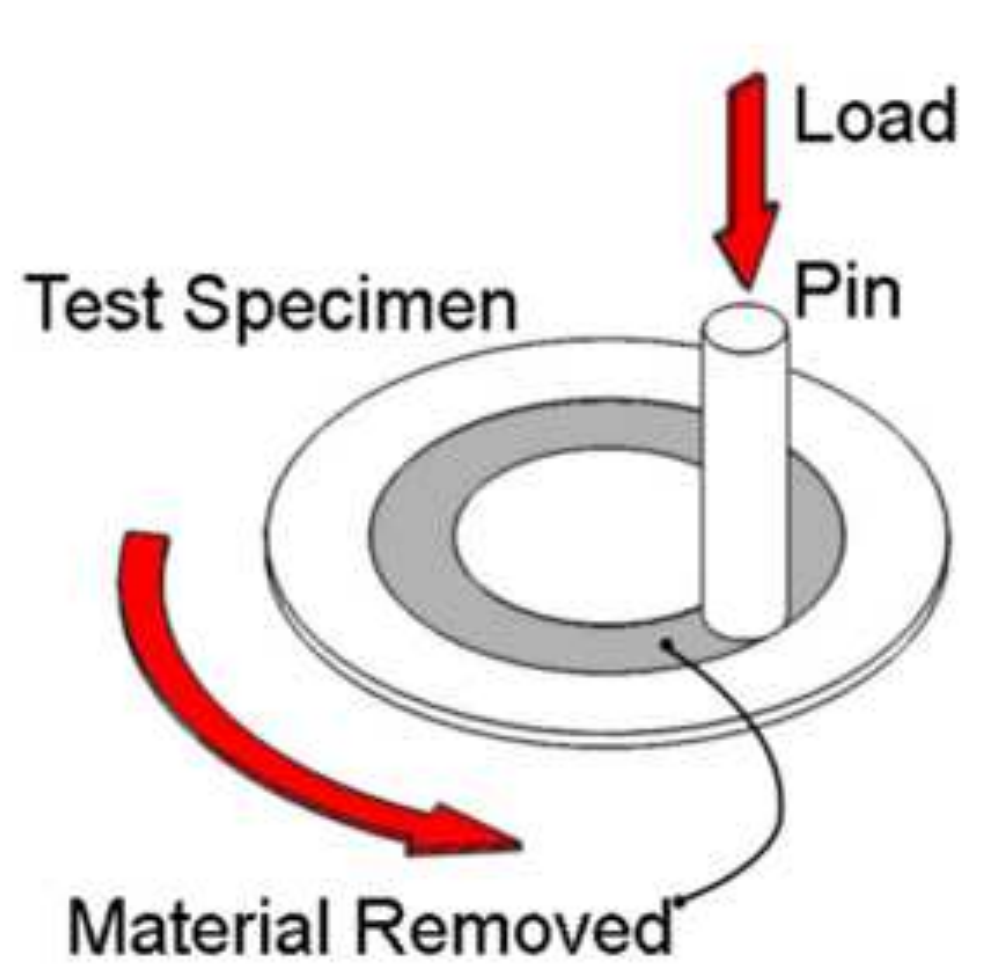

B

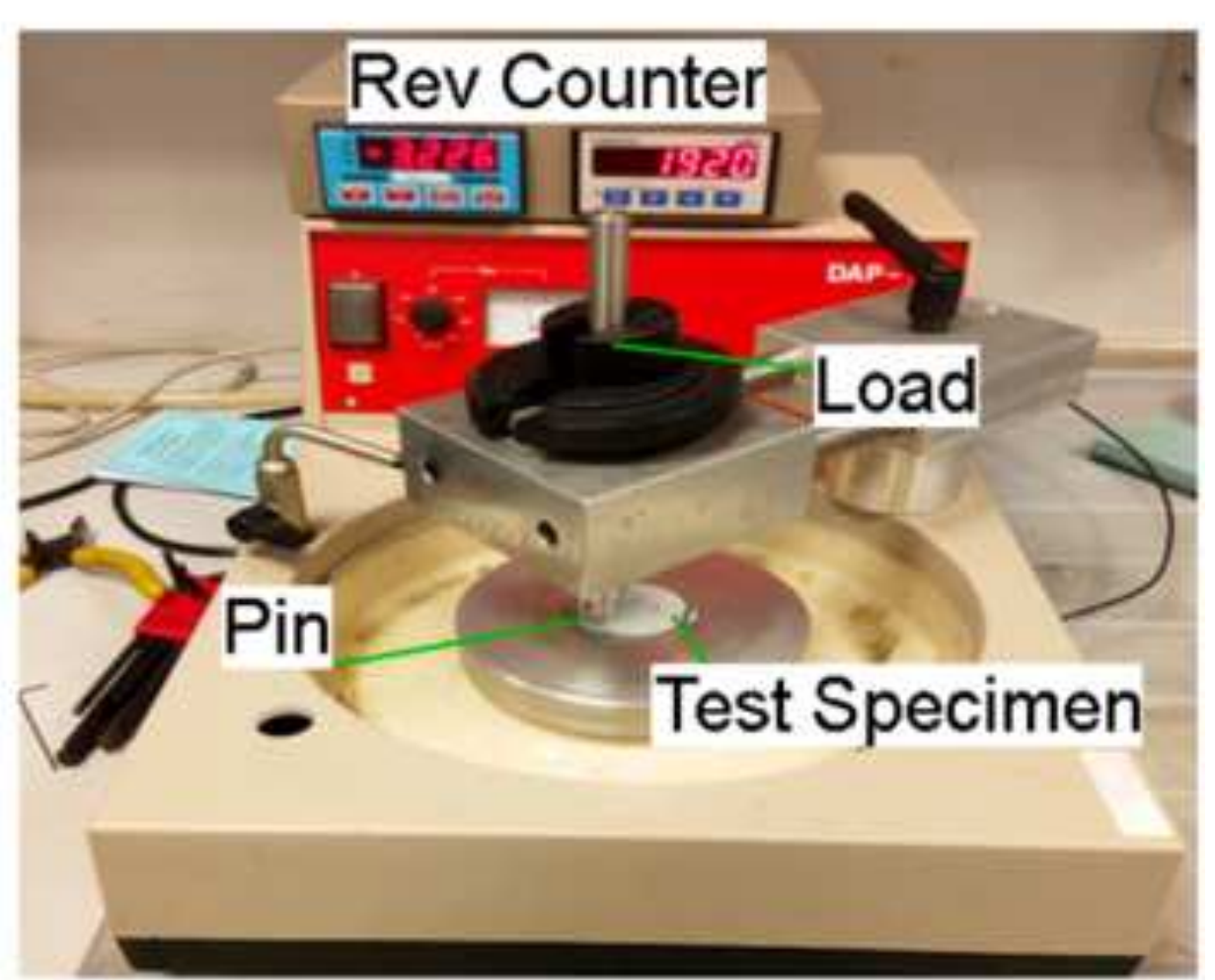




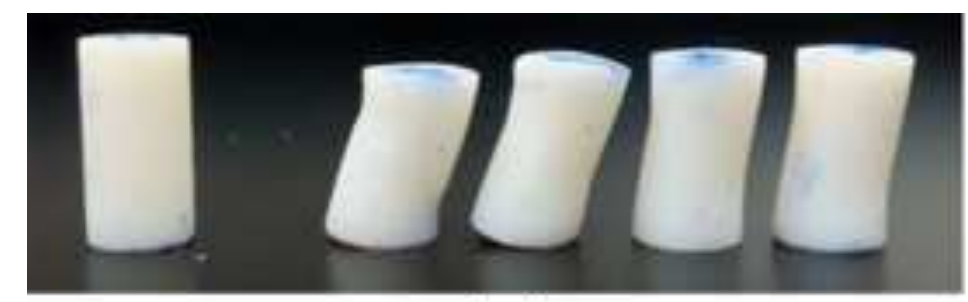

(a)

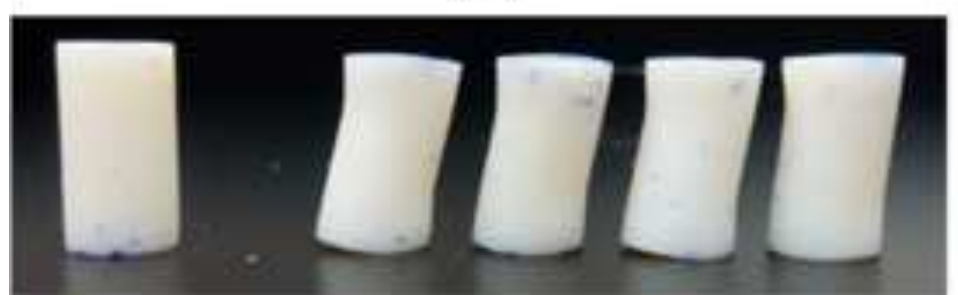

(c)

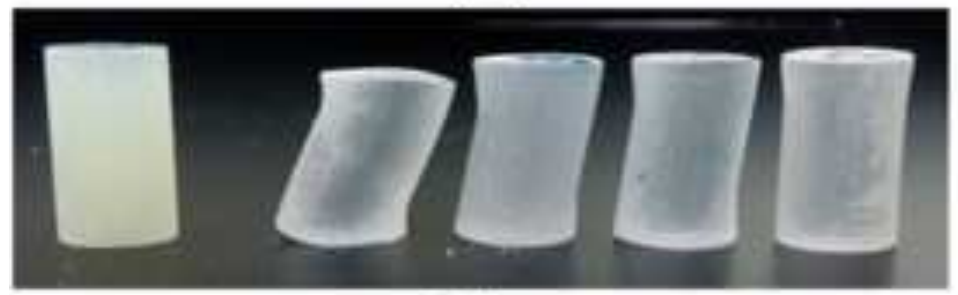

(e)

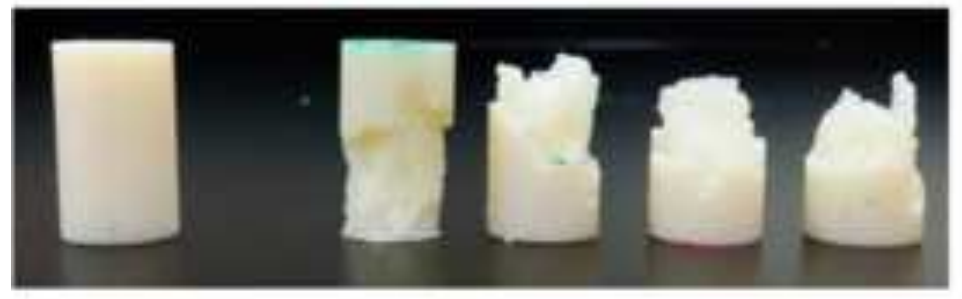

(g)

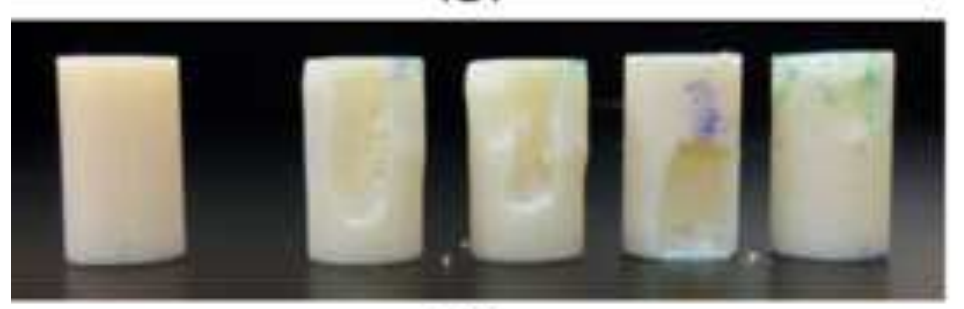

(i)

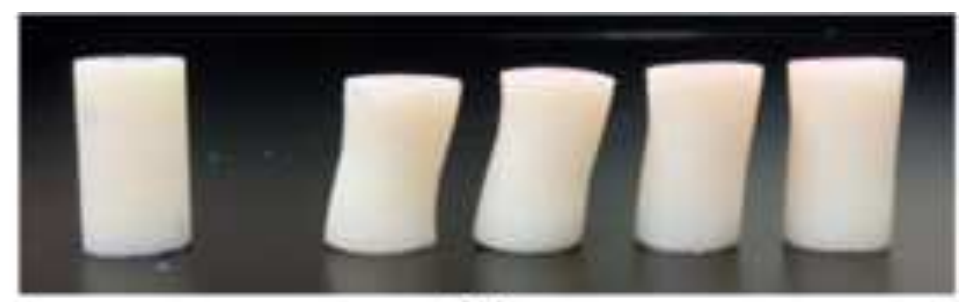

(b)

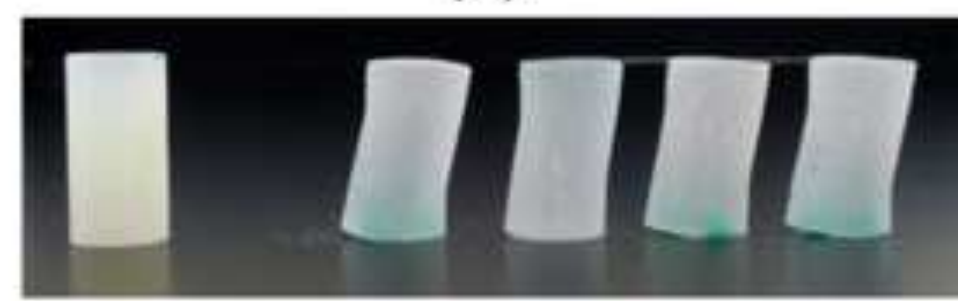

(d)

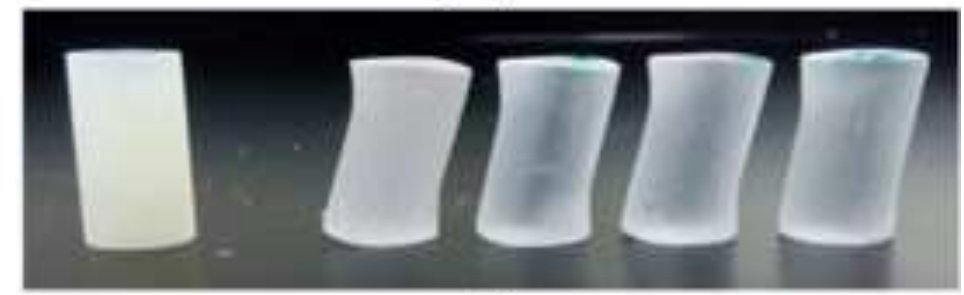

(f)

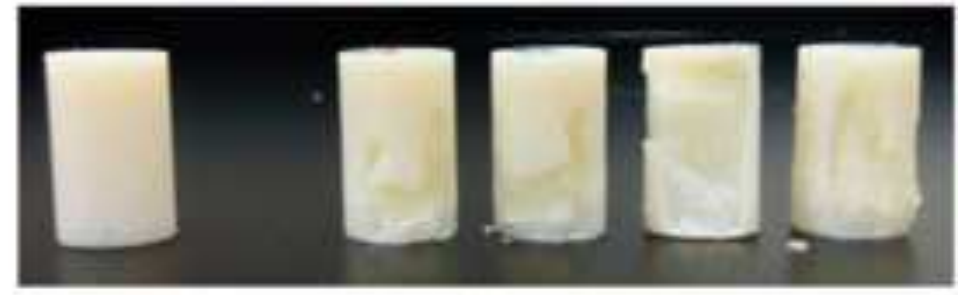

(h)

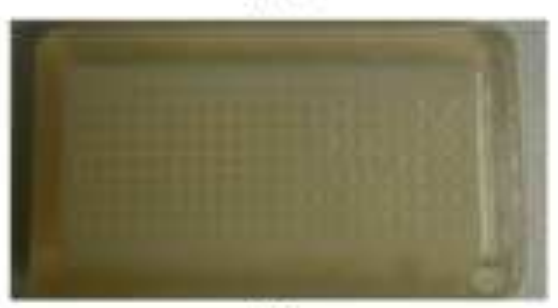

(j) 

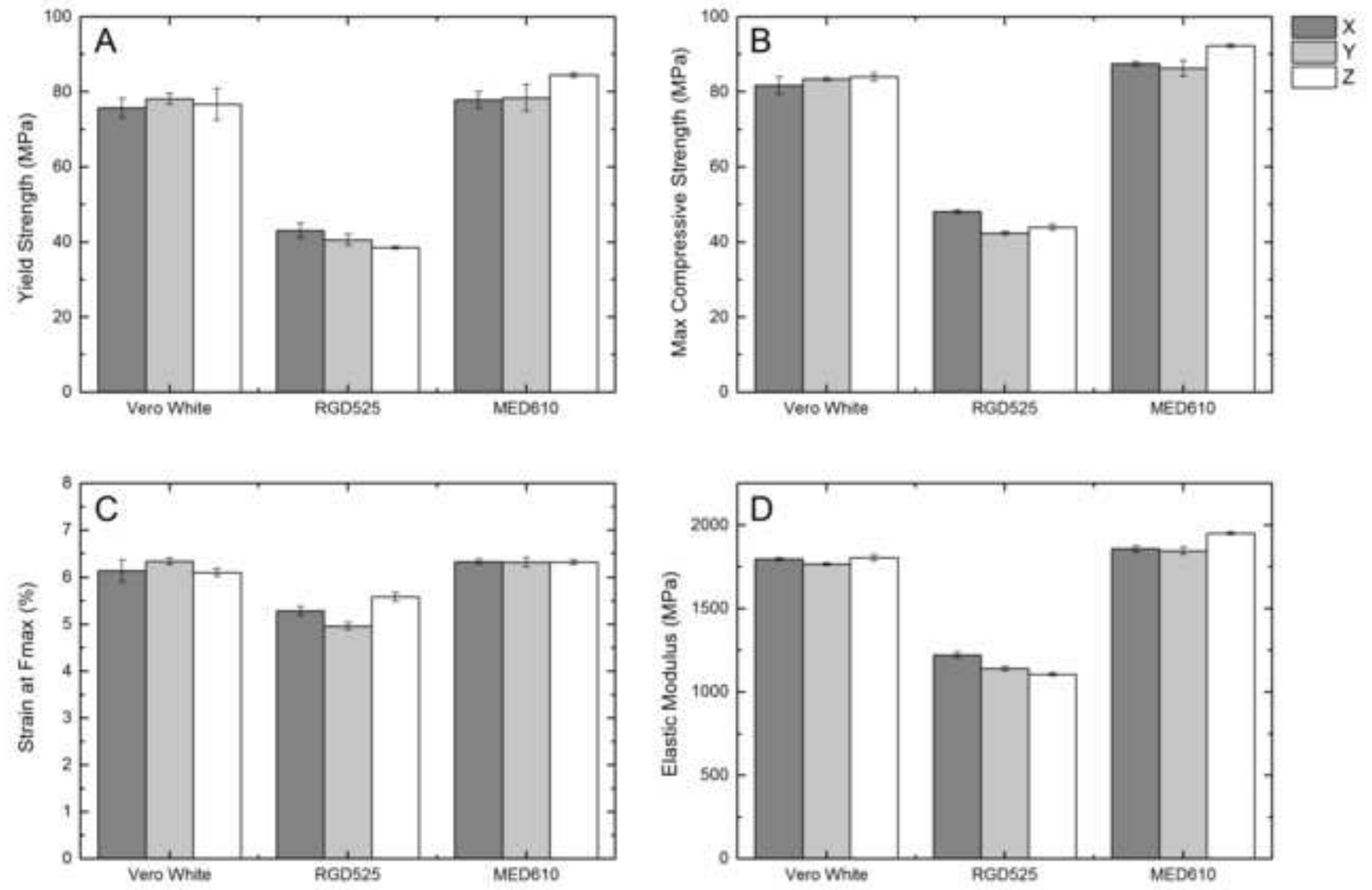


\section{Vero White}

\section{MED610}

\section{RGD525}

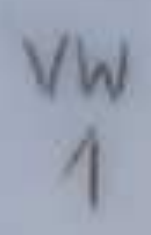

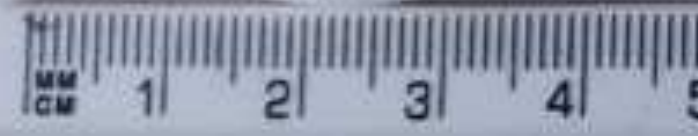
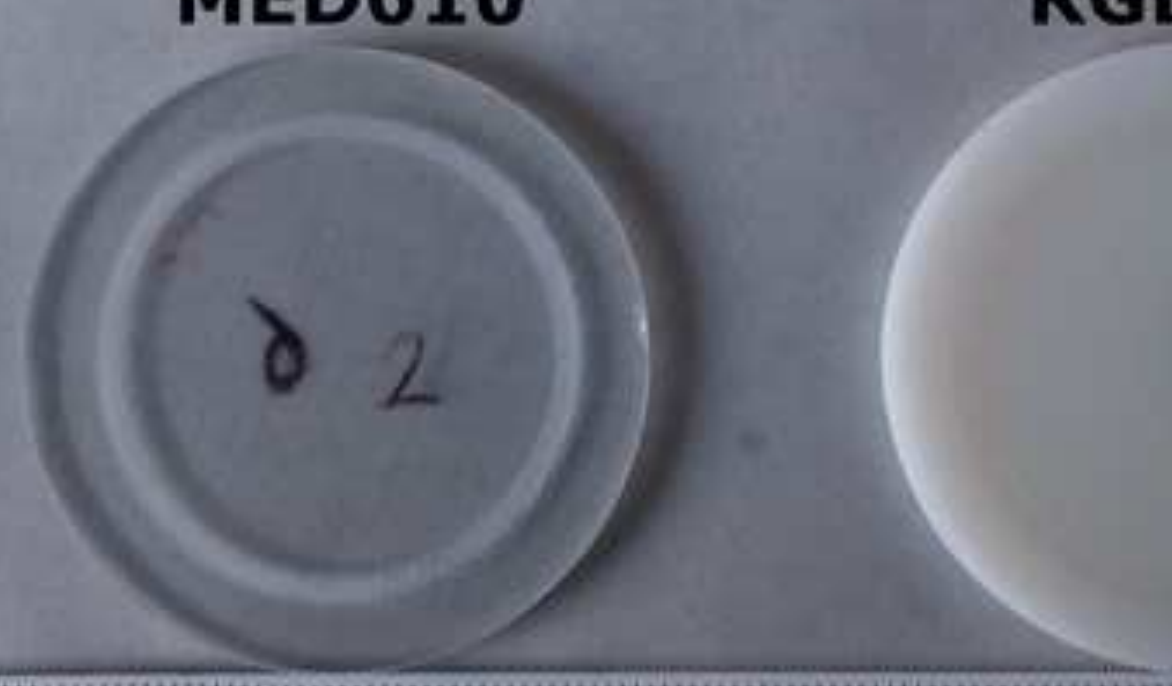

3

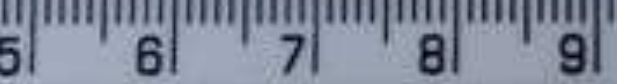

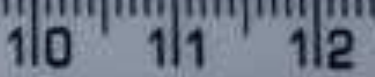

13

14

15

16 


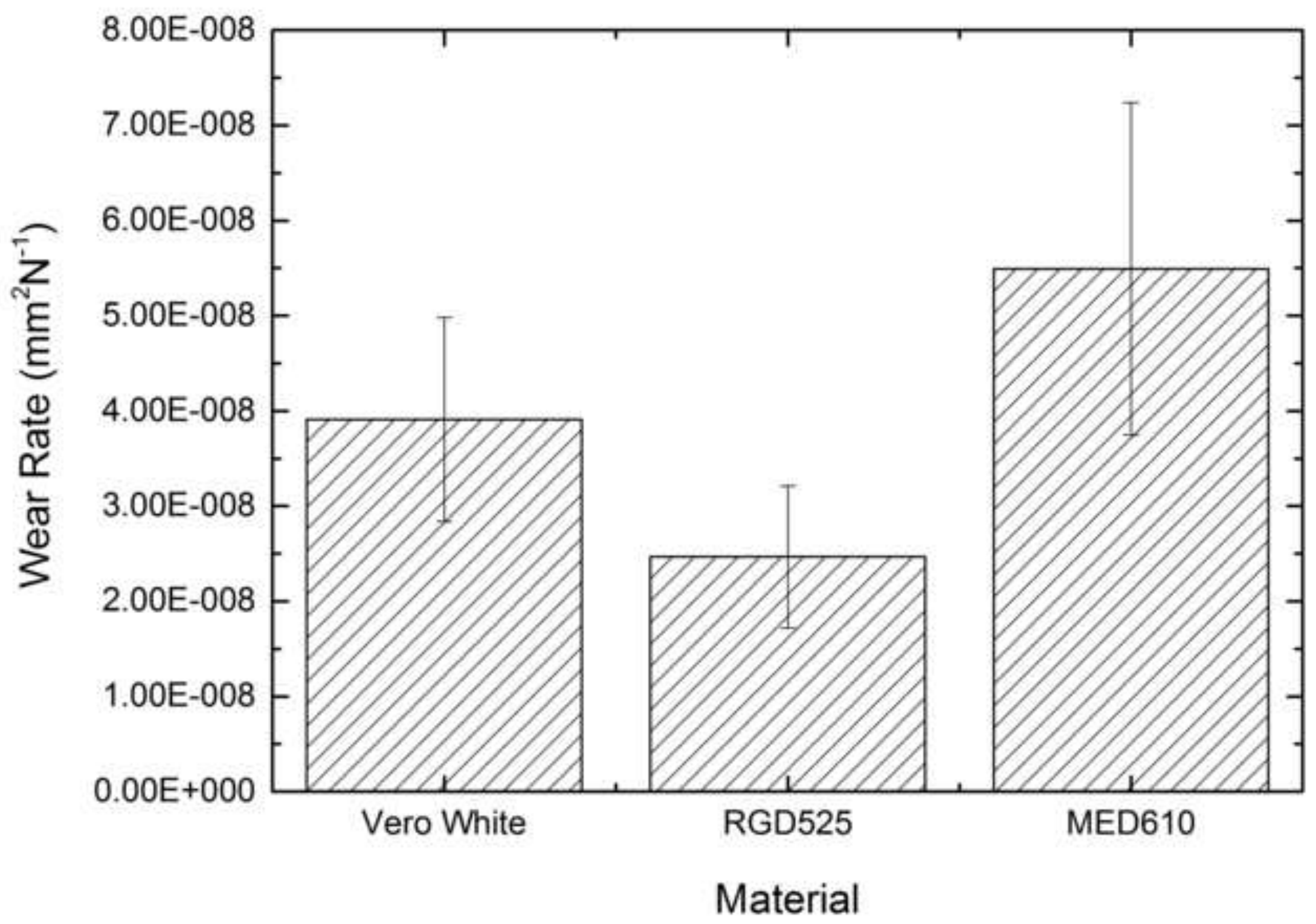

